\title{
Identification of essential language areas by combination of fMRI from different tasks using probabilistic independent component analysis
}

\author{
Yanmei Tie ${ }^{1}$, Ralph O. Suarez, Stephen Whalen, Isaiah H. Norton, Alexandra J. Golby
}

${ }^{1}$ Departments of Neurosurgery and Radiology, Brigham and Women's Hospital, Harvard Medical School, Boston, MA, USA. Correspondence should be addressed to Alexandra J. Globy (agolby@bwh.harvard.edu), Tel.: 617-525-6776.

Received August 5, 2008; revised October 14, 2008; accepted October 14, 2008

\begin{abstract}
Functional magnetic resonance imaging (fMRI) has been used to lateralize and localize language areas for pre-operative planning purposes. To identify the essential language areas from this kind of observation method, we propose an analysis strategy to combine fMRI data from two different tasks using probabilistic independent component analysis (PICA). The assumption is that the independent components separated by PICA identify the networks activated by both tasks. The results from a study of twelve normal subjects showed that a language-specific component was consistently identified, with the participating networks separated into different components. Compared with a model-based method, PICA's ability to capture the neural networks whose temporal activity may deviate from the task timing suggests that PICA may be more appropriate for analyzing language fMRI data with complex event-related paradigms, and may be particularly helpful for patient studies. This proposed strategy has the potential to improve the correlation between fMRI and invasive techniques which can demonstrate essential areas and which remain the clinical gold standard.
\end{abstract}

Keywords: fMRI, probabilistic independent component analysis (PICA), language mapping, event-related paradigm

\section{INTRODUCTION}

The purpose of pre-surgical language mapping is to lateralize and localize critical language areas for neurosurgical planning when the patient's lesion is located in or close to language areas. In addition to invasive language mapping techniques (e.g., intracarotid amytal test (IAT), and intra-operative electric cortical stimulation (ECS)), pre-operative functional magnetic resonance imaging
(fMRI) based on language tasks has been used to determine the language-dominant hemisphere [1] and provide spatial relationships between brain lesions and language areas [2]. Although fMRI has the advantages of non-invasiveness, pre-operative availability, repeatability, and less time and cost, it has several shortcomings for language mapping applications [3]. First, compared to the conventional inhibition methods that are able to demonstrate essential areas, fMRI is an observation method which thus demonstrates numerous areas involved in the language tasks, but cannot demonstrate the necessity of those areas in language function. Second, language fMRI generally uses silent tasks due to the motion artifact resulting from vocalizing responses, which complicates comparisons with the clinical gold standard tests that use overt responses.

To try to address these problems, we applied a data-driven method, probabilistic independent component analysis (PICA) [4], to fMRI data from two language tasks. First, we propose an analysis strategy to examine activations during two different tasks in an effort to identify the essential language areas. Second, we investigate the performance of PICA in extracting language-related components from vocalized language fMRI data that are contaminated by motion artifact and background noise.

Independent component analysis (ICA) has been applied to fMRI data to extract statistically independent features $[5,6]$. It has been shown that ICA can be used as a complementary tool to the conventional general linear model (GLM) method in improving the sensitivity and specificity of fMRI language mapping [7]. PICA is an extension of the classical noise-free ICA model [5], assuming that the data are confounded by additive Gaussian noise [4]. It was proposed to address the overfitting problem and make the statistical significance testing feasible for the analysis of fMRI data. PICA has been applied in several studies, including the investigation of the neural dynamics of default-mode networks and event segmentation in music [8-11].

\section{MATERIALS}




\subsection{Subjects and Image Acquisition}

The protocol was approved by the Partner's Institutional Review Board. Twelve right-handed native English speaking healthy subjects participated (7 men, 5 women, mean age $=30.6 \pm 6.5$ years, range $20-43$ years). One right-handed patient with focal cortical displasia (female, 35 years old) was also studied. All subjects provided written informed consent.

MR images were obtained using a 3.0 Tesla scanner (Signa scanner, GE Medical System, Milwaukee, WI, USA). A single-shot gradient-echo echo-planar imaging (EPI) was used to acquire blood-oxygen-level dependent (BOLD) functional images $(\mathrm{TR}=1000 \mathrm{~ms}$, $\mathrm{TE}=29 \mathrm{~ms}$, flip angle $=68^{\circ}, \mathrm{FOV}=24 \mathrm{~cm}$, dimension $=64 \times 64 \mathrm{x}$ 16 , slice gap $=0 \mathrm{~mm}$, voxel size $=3.75 \times 3.75 \times 5 \mathrm{~mm}^{3}$ ) using a quadrature head coil. In each image volume, 16 axial slices were acquired using ascending interleaved scanning sequence. A volumetric T1-weighted magnetization prepared rapid gradient echo (MPRAGE) acquisition $($ dimension $=256 \times 256 \times 124$, voxel size $=1 \times 1 \times$ $1.3 \mathrm{~mm}^{3}$ ) was performed to provide a high-resolution anatomic reference frame for subsequent overlay of functional activation maps.

The patient's functional images were acquired using different parameters $(\mathrm{TR}=2000 \mathrm{~ms}, \mathrm{TE}=40 \mathrm{~ms}$, flip angle $=90^{\circ}, \mathrm{FOV}=24 \mathrm{~cm}$, dimension $=128 \times 128 \times 28$, slice gap $=0 \mathrm{~mm}$, voxel size $=1.88 \times 1.88 \times 4 \mathrm{~mm}^{3}$, ascending interleaved scanning sequence). High-resolution T2-weighted gradient-echo MR images (dimension $=512 \times 512 \times 91$, voxel size $=0.5 \times 0.5 \times$ $1.5 \mathrm{~mm}^{3}$ ) were acquired to provide background structural images for the patient's activation maps.

\subsection{Behavioral Paradigm}

Subjects performed two language tasks with vocalized responses: antonym-generation (AG), and noun categorization (NC). In the AG task, subjects were asked to speak the antonym of a word stimulus presented visually through MR-compatible video goggles (Resonance Technology, Los Angeles, CA, USA). In the NC task, subjects were asked to state whether the word stimulus referred to either a living ("alive", e.g., a dog) or non-living ("not alive”, e.g., a chair) object. Subject vocalizations were transmitted by an intercom system (Avotec Inc., Stuart, FL, USA) to an investigator in the MRI scanner control room who counted the number of incorrect or omitted responses in order to verify satisfactory task performance. Subjects were instructed to verbalize responses with minimal movement of their head, jaw, or lips. During the time period between visual stimuli, subjects were asked to relax and look at a crosshair shown in the center of the visual field.

The language tasks were implemented as a rapid-presentation, event-related fMRI paradigm with a jittered inter-stimulus-interval (ISI = $8.3 \pm 5.1 \mathrm{sec}$ ), lasting 7 min $20 \mathrm{sec}$ (including a 10-sec pre-stimulus period acquired to allow stabilization of the BOLD signal, excluded from analysis). A total of 50 word stimuli were delivered during each task, and each word was shown for 2 sec. The fMRI paradigms of the patient study lasted 5 min 20 sec (including a 10-sec pre-stimulus period), delivering 34 words in the AG task and 39 words in the NC task). The order and exact timing for delivery of word stimuli was based on a stochastic design intended to maximize the statistical significance of the fMRI paradigm, and minimize subject's expectation and habituation effects. Stimuli event scheduling was performed using the Optseq2 software package (NMR Center, Massachusetts General Hospital, MA, USA). Stimulus paradigms were implemented using Presentation software package (Version 9.70, Neurobehavioral Systems Inc., Davis, CA, USA).

\section{METHODS}

\subsection{Concatenation of Two Tasks and Data Pre-processing}

FMRI data from two tasks were concatenated in time by putting the NC task data at the end of the AG task data (Figure 1). Thus the total data set of each subject was 860 volumes (430 volumes for each task). Then we used the Statistical Parametric Mapping software package (SPM2, Wellcome Department of Cognitive Neurology, London, UK) to perform motion correction by realigning the fMRI images to the first functional image.

The data were then sent to the Multivariate Exploratory Linear Optimized Decomposition into Independent Components (MELODIC, Version 3.05) module of FMRIB's Software Library (FSL, Version 4.0, Oxford Center for Functional Magnetic Resonance Imaging of the Brain, University of Oxford, Oxford, UK) for PICA analysis [4]. Before the PICA procedure, the following steps were applied to the input data file: masking of non-brain voxels by an intensity thresholding at $10 \%$; high-pass temporal filtering to remove low-frequency drifts with cut-off period of $128 \mathrm{sec}$; and voxel-wise de-meaning and variance normalization of the data.

\subsection{Probabilistic ICA of fMRI Data}

We applied the PICA technique proposed by Beckmann and Smith [4] to analyze the concatenated fMRI data. The fMRI signal $(X)$ is assumed to be generated from a linear mixing process of the independent non-Gaussian sources $(S)$ by a mixing matrix $(A)$, and corrupted by additive Gaussian noise $(\eta)$ :

$$
X=A S+\eta \text {. }
$$

In the PICA model (1), $X$ is a $p \times n$ matrix denoting $p$ volumes ( $p=860$ volumes for this study) of $n$ voxels fMRI data, $S$ is a $q \times n$ matrix denoting $q$ non-Gaussian sources (i.e., independent components, ICs), and $A$ is a $p$ $\times q$ mixing matrix.

First, the number of ICs $(q)$ was estimated using the Laplace approximation to the Bayesian evidence of the model order [4]. There were $95 \sim 166$ components estimated for each subject (mean \pm STD $=122 \pm 20$, across 


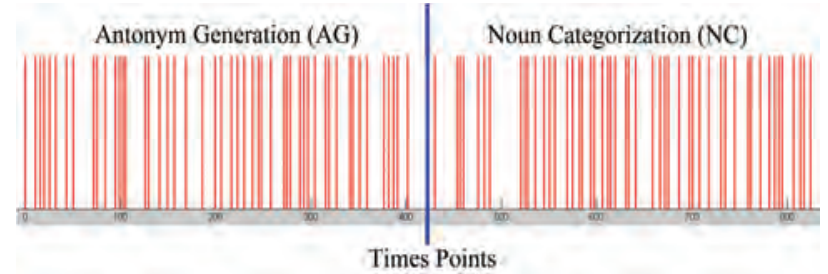

Figure 1. Concatenation of two tasks fMRI data. Red bars: word stimuli onsets.
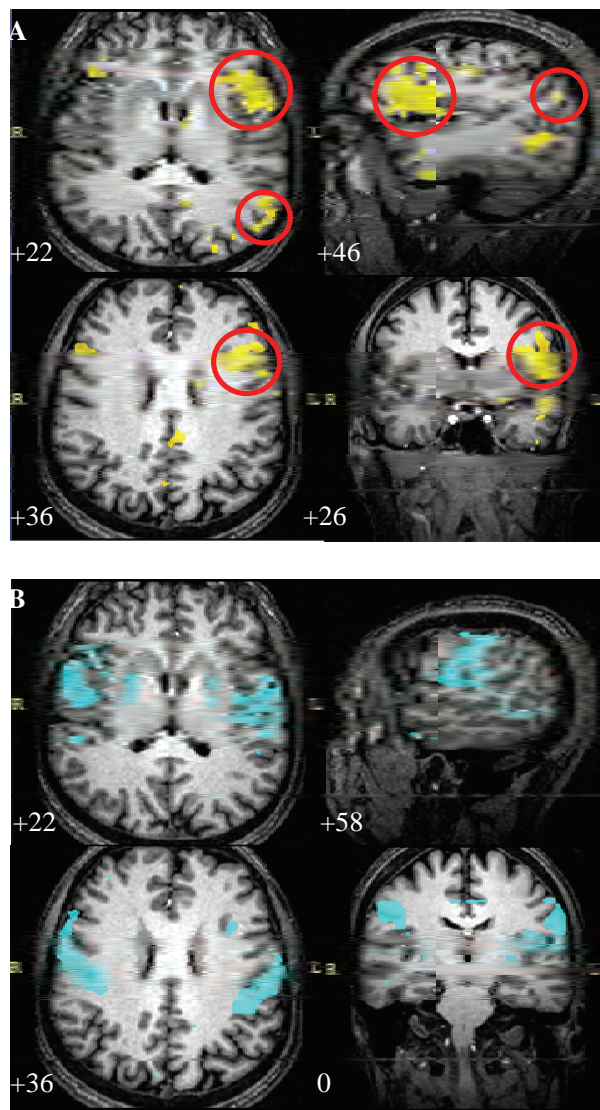

Figure 2. Spatial maps of (A) "language COI", and (B) "motor COI" of an example subject estimated by the PICA analysis (posterior probability thresholded at $p>0.5$ ).

subjects). The fMRI data were then reduced to $q$ dimensions by principal component analysis (PCA) and decomposed into $q$ spatially independent components by the fastICA algorithm [12]. A de-mixing matrix $W$ was found to generate:

$$
\hat{S}=W X,
$$

where $\hat{\mathrm{S}}$ is a good approximation to the sources.

Then the estimated component maps were divided by the standard deviation of the Gaussian noise to generate z-score maps, and sequentially thresholded at a posterior probability $p>0.5$, by fitting a Gaussian/Gamma mixture model to the histogram of intensity values [4].

\subsection{Identification of Components of Interest}

We used a two-step process to identify the components
Table 1. Ranking results of COIs based on IC time course's average power in frequency range of less than $0.1 \mathrm{~Hz}$.

\begin{tabular}{|l|l|l|}
\hline Subject & Language COI & Motor COI \\
\hline 1 & $4^{\text {th }}$ & $3^{\text {rd }}$ \\
\hline 2 & $8^{\text {th }}$ & $4^{\text {th }}$ \\
\hline 3 & $1^{\text {st }}$ & $2^{\text {nd }}$ \\
\hline 4 & $11^{\text {th }}$ & $10^{\text {th }}$ \\
\hline 5 & $14^{\text {th }}$ & $9^{\text {th }}$ \\
\hline 6 & $5^{\text {th }}$ & $4^{\text {th }}$ \\
\hline 7 & $5^{\text {th }}$ & $6^{\text {th }}$ \\
\hline 8 & $1^{\text {st }}$ & $11^{\text {th }}$ \\
\hline 9 & $6^{\text {th }}$ & $5^{\text {th }}$ \\
\hline 10 & $23^{\text {rd }}$ & $25^{\text {th }}$ \\
\hline 11 & $3^{\text {rd }}$ & $8^{\text {th }}$ \\
\hline 12 & $1^{\text {st }}$ & $9^{\text {th }}$ \\
\hline
\end{tabular}

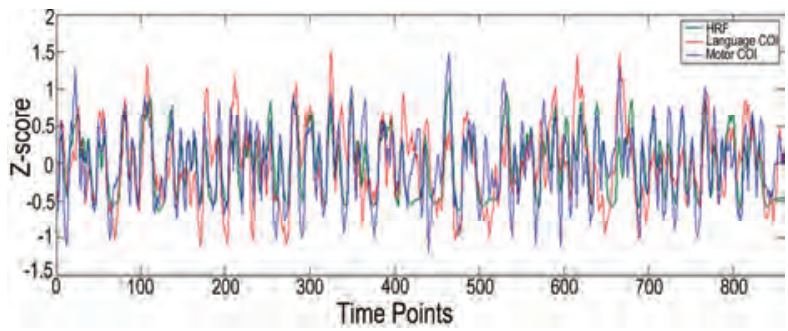

Figure 3. Time courses of "language COI" (red) and "motor COI" (blue) averaged across subjects. The green plot is the expected HRF model.

of interest (COIs) from the PICA output of each subject's data. First, the power spectrum density of each component's time course was estimated by periodogram spectral estimation. Then the average power was calculated for frequency less than $0.1 \mathrm{~Hz}$, and ranked in descending order. This frequency range was selected based on the power spectrum of the expected hemodynamic response function (HRF), which was in low-frequency range $(<0.1 \mathrm{~Hz})$. Next, the components' spatial maps were visually inspected in the order determined by the previous step to identify the components with activation in the putative language areas, and other areas of interest.

\subsection{GLM Analysis of fMRI Data}

For comparison purposes the pre-processed data were submitted to SPM2 for conventional GLM analysis. The basis function consisted of the canonical HRF model with temporal and dispersion derivatives. Run-specific responses were modeled in an event-related design [13] by convolving a series of Dirac's delta function, each representing a stimulus event onset, with the basis function. After GLM, the $t$ maps were fitted to a Gaussian/Gamma mixture model and thresholded at a posterior probability $p>0.5$ in order to be comparable with the PICA results.

\section{RESULTS}




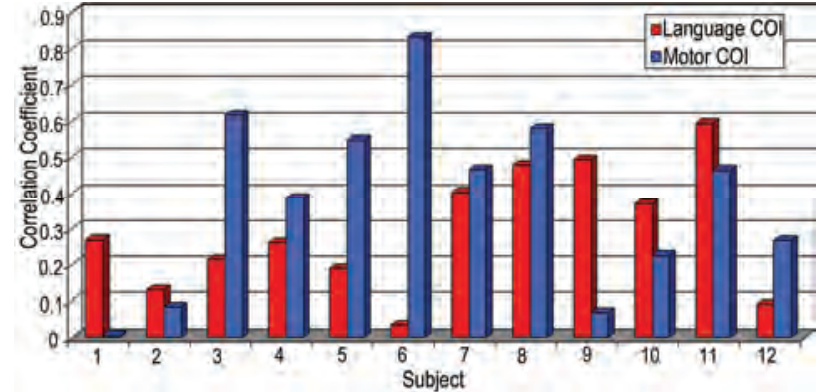

Figure 4. Temporal correlation coefficients between the identified COls' time courses and the expected HRF model for each subject (red bars: language COIs; blue bars: motor COIs).

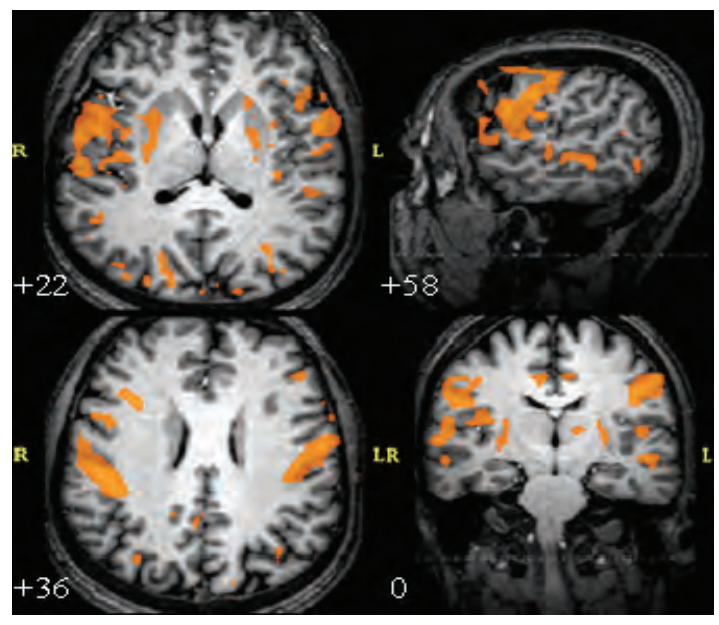

Figure 5. Spatial maps of GLM analysis of the same example subject as in Fig. 2 (posterior probability thresholded at $p$ $>0.5)$.

\subsection{Spatial Maps of Identified Language and Motor COIs}

For each subject, among an average of 122 components estimated by PICA, one component was consistently identified with activations in the left frontal and temporal lobes, primarily in the putative language areas (inferior frontal gyrus, superior temporal gyrus, and supramarginal gyrus), and pre-motor area (PMA, middle frontal gyrus). This component was named "language COI”. Figure 2A shows the spatial maps of the language COI of an example subject (activations in the putative language areas and PMA are highlighted by red circles). Another component was identified with bilateral activations mainly in the primary face motor areas (precentral gyrus), which was named "motor COI". Figure 2B shows the spatial maps of the motor COI of the same subject.

The ranking results of the language and motor COIs based on the average power of their time courses are listed in Table 1 for each subject. It demonstrated that these COIs were ranked within the first 15 ICs (except for one subject (\#10), whose language COI ranked the 23rd, and motor COI ranked the 25th).

\subsection{Time Courses of Identified COIs}
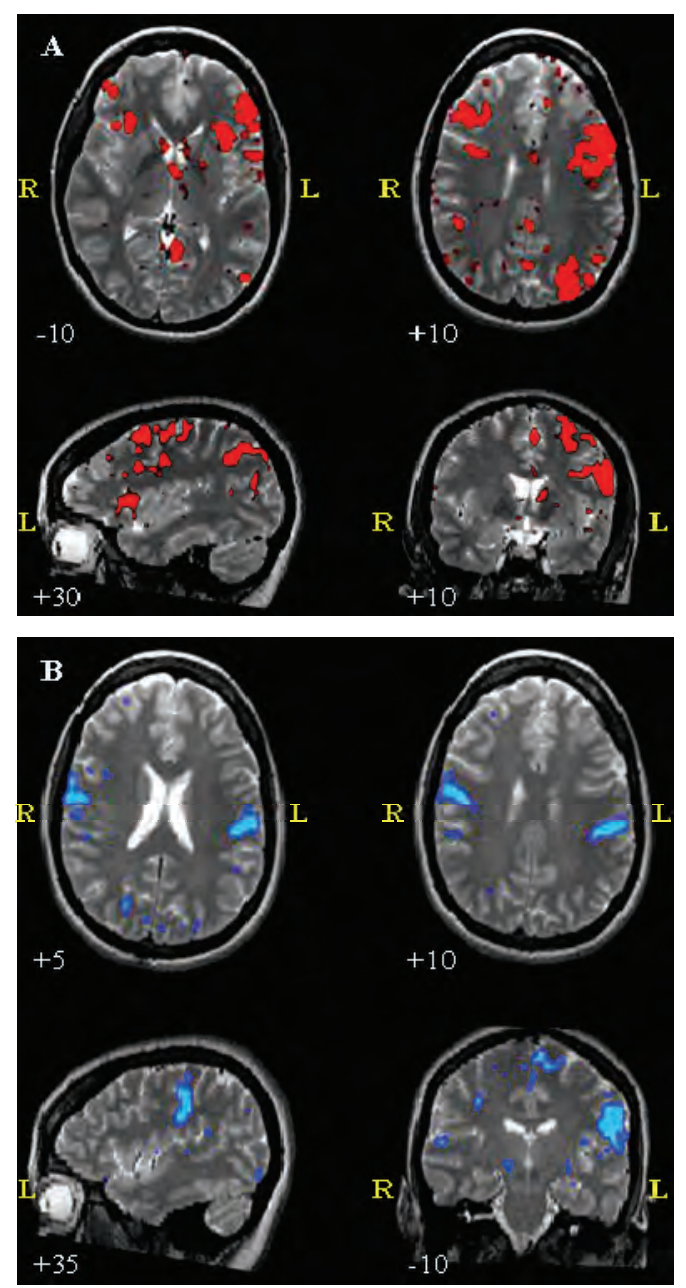

Figure 6. Spatial maps of (A) "language COI", and (B) "motor COI" of the patient estimated by the PICA analysis (posterior probability thresholded at $p>0.5$ ).

Figure 3 shows the time courses of the language (red) and motor (blue) COIs averaged across all subjects. The expected HRF model is also shown (green).

Figure 4 shows the results of temporal correlation analysis between the identified COIs' time courses and the expected HRF mode for each subject. It demonstrated that the time courses of the motor COIs of 7 subjects correlated more closely with the expected HRF than that of the language COIs. The correlation coefficients are $0.30 \pm 0.17$ (mean \pm STD, across subjects) for the language COIs, and $0.38 \pm 0.25$ for the motor COIs.

\subsection{Comparison of PICA and GLM Results}

Figure 5 shows the spatial maps of the GLM results of the same example subject. Compared with the PICA maps, the GLM maps identified activation patterns very similar to that of the motor COI (Figure 2B), with bilateral activations mainly in the primary face motor areas. The GLM maps showed weak activations in the putative language areas.

\subsection{Results of Patient Data}




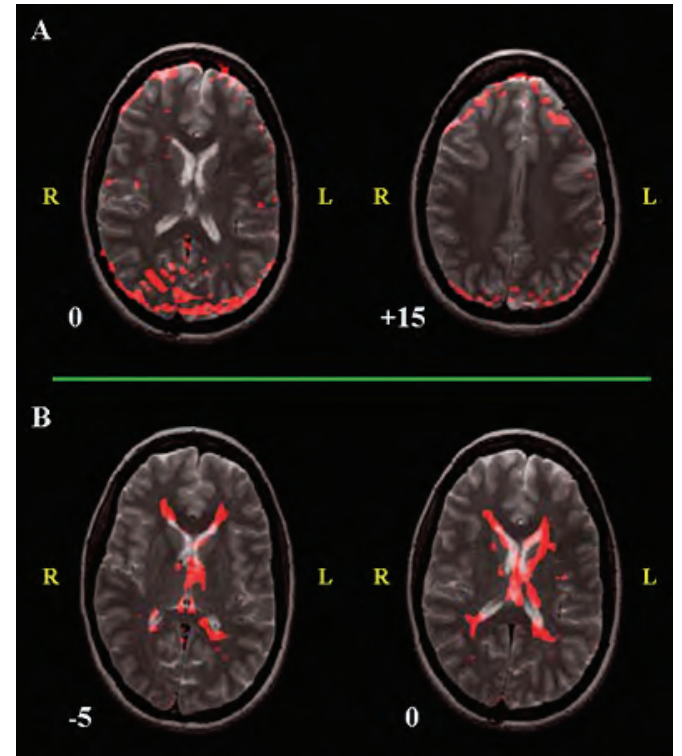

Figure 7. Two noise components showing signals from (A) the brain edge; and (B) the ventricle system.

The spatial maps of the language and motor COIs resulting from PICA analysis of the patient data are shown in Figure 6. The "language COI" (Figure 6A) showed significant activations in the left inferior frontal gyrus and left PMA. The "motor COI" (Figure 6B) shows bilateral activations in the primary face motor areas.Temporal correlation analysis indicated that the language COI correlated poorly with the task timing ( $r=$ $0.15, p<0.01$ ), while the motor COI correlated highly with the task timing $(r=0.45, p<0.0001)$. Using the same pre-processed data, GLM generated maps (not shown) revealed activations mainly in the bilateral motor area, but almost no activations in the putative language areas.

Figure 7 shows two noise components indicating signals from the edge of the brain (due to motion artifact, Figure 7A) and the ventricle system (Figure 7B).

\section{DISCUSSION}

In this study, we propose a new analysis strategy using PICA and combining fMRI data from different language tasks to better identify the essential language areas for pre-operative language mapping. A component was consistently identified in the putative language areas, suggesting that the underlying system was essential to both tasks. Compared with the conventional GLM method, PICA indicated better performance in extracting language activations, and separating noise into different components.

It is clinically useful to demonstrate brain regions critical for language function so that they may be avoided during resection [3]. Whereas, clinical tests which block neural activity (IAT and ECS) are able to demonstrate the necessity of particular areas for language function, fMRI maps may include non-language-specific areas that participate in the task
[7]. To try to demonstrate language-specific areas, we concatenated the fMRI data from two different tasks in time, and applied PICA to estimate the spatially independent components from the data. The underlying assumption is that the networks revealed by PICA are activated by both tasks, which are more likely to be essential language areas. The results of all subjects consistently identified a network in the putative language areas and the ipsilateral PMA. Another network was found in the bilateral face motor areas, indicating the participation of these areas in overt language production.

The temporal correlation analysis on the time courses of the "language COIs" showed a relatively low correlation with the expected HRF model, while the "motor COIs" correlated more closely with the HRF model. This indicated that the temporal profile of the language activation may differ from the task timing, while the motor activation followed the task very well. Since the GLM method is based on the expected HRF model, and therefore able to identify voxels whose time courses correlate highly with the paradigm, this may explain the observation that the GLM identified very similar activation patterns to the "motor COIs", but did not show significant activations in the language areas. PICA's ability to capture the neural networks whose temporal activities may deviate from the time course of the paradigm suggests that the data-driven method may be well-suited to analysis of complex event-related language fMRI. The data-driven method could be particularly useful in analyzing patient data, since the lesions adjacent to the language areas may result in alterations in the BOLD response [14], and patients may have difficulty with task performance causing altered timing of the cognitive process relative to the model.

To identify components of interest from a large number of separated components remains a practical challenge for the ICA technique, and methods have been proposed based on spatial, temporal, and spectral criteria $[5-7,15]$. In this study, we ranked the ICs based on their time courses' average power in the frequency range of less than $0.1 \mathrm{~Hz}$. This frequency range was chosen based on the power spectrum of the expected HRF model. The language and motor COIs were among the highly ranked ICs, which confirmed the effectiveness of this selection criterion. Among other highly ranked ICs were default-mode networks as shown in [8], and low frequency head motion artifact.

Vocalized event-related language paradigms offer an advantage of more closely simulating natural language performance. However, the motion artifact resulting from vocalizing responses may lead to contamination in the statistical maps. PICA has the ability to separate signals from motion artifact, noise, and physiological effects, into different components, and therefore may be particularly applicable to vocalized language fMRI data.

This proposed combination strategy can be extended to fMRI data from multiple tasks. To improve the effectiveness of this strategy, the different tasks should be 
selected in an optimal way. In this study, the AG task focused on both the receptive and expressive aspects of language function, and the NC task was more involved in the receptive aspect. Future work will be directed at the optimal combination of task paradigms, as well as validation of this approach against invasive testing in patients.

\section{CONCLUSION}

We propose a new analysis strategy to identify essential language areas by combining fMRI data from two different tasks. We applied a data-driven method, PICA, based on the assumption that the separated spatially independent networks were activated by both tasks. The results show that using this approach, the language component was consistently identified and separated from the participating networks. This approach compares favorably with GLM for complex event-related language paradigms, and may be particularly helpful for patient studies for pre-operative language mapping.

\section{ACKNOWLEDGEMENT}

This study is supported by NIH K08 NS048063, NIH-NCRR U41 RR019703, and The Brain Science Foundation.

\section{REFERENCES}

[1] J.R. Binder, S.J. Swanson, T.A. Hammeke, G.L. Morris, W.M. Mueller, M. Fischer, S. Benbadis, J.A. Frost, S.M. Rao, and V.M. Haughton, (1996) "Determination of language dominance using functional MRI: a comparison with the Wada test," Neurology, vol. 46, 978-984.

[2] C. Stippich, N. Rapps, J. Dreyhaupt, A. Durst, B. Kress, E. Nenning, V.M. Tronnier, and K. Sartor, (2007) "Localizing and lateralizing language in partients with brain tumors: feasibilty of routine preoperative functional MR imaging in 81 consecutive patients," Radiology, vol. 243, 828-836.

[3] S. Tharin and A. Golby, (2007) "Functional brain mapping and its applications to neurosurgery,” Neurosurgery, vol. 60, 185-201.

[4] C.F. Beckmann and S.M. Smith, (2004) "Probabilistic independent component analysis for functional magnetic resonance imaging," IEEE Trans. Med. Imaging, vol. 23, 137-152.

[5] M.J. McKeown, S. Makeig, G.G. Brown, T.P. Jung, S.S. Kindermann, A.J. Bell, and T.J. Sejnowski, (1998) "Analysis of fMRI data by blind separation into independent spatial components," Hum. Brain Mapp., vol. 6, 160-188.

[6] V.D. Calhoun, T. Adali, G.D. Pearlson, and J.J. Pekar, (2001) “A method for making group inferences from functional MRI data using independent component analysis," Hum. Brain Mapp., vol. $14,140-151$.

[7] Y. Tie, S. Whalen, R.O. Suarez, and A.J. Golby, (2008) “Group independent component analysis of language fMRI from word generation tasks," Neuroimage, vol. 42, 1214-1225.

[8] M.D. Greicius, G. Srivastava, A.L., Reiss, and V. Menon, (2004) "Default-mode network activity distinguishes Alzheimer's disease from healthy aging: Evidence form functional MRI," Proc. Natl. Acad. Sci. U.S.A., vol. 101, 4637-4642.

[9] C.F. Beckmann, M. DeLuca, J.T. Devlin, and S.M. Smith, (2005) "Investigations into resting-state connectivity using independent component analysis,” Phil. Trans. R. Soc. B, vol. 360, 1001-1013.

[10] M. Fukunaga, S.G. Horovitz, P. Van Gelderen, J.A. de Zwart, J.M. Jansma, V.N. Ikonomidou, R. Chu, R.H.R. Deckers, D.A. Leopold, and J.H. Duyn, (2006) "Large-amplitude, spatially correlated fluctuations in BOLD fMRI signals during extended rest and early sleep stages,” Magn. Reson. Imaging, vol. 24, 979-992.

[11] D. Sridharan, D. J. Levitin, C.H. Chafe, J. Berger, and V. Menon, (2007) "Neural dynamics of event segmentation in music: Converging evidence for dissociable ventral and dorsal networks," Neuron, vol. 55, 521-532.

[12] A. Hyvarinen, (1999) "Fast and robust fixed-point algorithms for independent component analysis," IEEE Trans. Neural. Netw., vol. $10,626-634$.

[13] K.J. Friston, P. Fletcher, O. Josephs, A. Holmes, M.D. Rugg, and R. Turner, (1998) "Event-related fMRI: characterizing differential responses," Neuroimage, vol. 7, 30-40.

[14] A.I. Holodny, M. Schulder, W.C. Liu, J. Wolko, J.A. Maldjian, and A.J. Kalnin, (2000)“The effect of brain tumors on BOLD functional MR imaging activation in the adjacent motor cortex: implications for image-guided neurosurgery," AJNR Am. J. Neuroradiol., vol. 21, 1415-1422.

[15] C.H. Moritz, B.P. Rogers, and M.E. Meyerand, (2003) "Power spectrum ranked independent component analysis of a periodic fMRI complex motor paradigm,” Hum. Brain Mapp, vol. 18, 111-122. 